\title{
Percepção de pessoas com hipertensão arterial sobre aspectos que influenciam a adesão ao tratamento
}

Perception of people with arterial hypertension about aspects that influence treatment adherence Percepción de las personas con hipertensión arterial sobre aspectos que influyen en la adherencia al tratamento

\section{Paulo Roberto de Oliveira Miranda ${ }^{\mathrm{I}}$, Débora de Oliveira Sacramento ${ }^{\mathrm{II}}$, Flávia Batista Barbosa de Sá Diaz ${ }^{\mathrm{III}}$, Luana Vieira Toledo ${ }^{\mathrm{IV}}$, Renan Sallazar Ferreira Pereira ${ }^{\mathrm{V}}$, Katiusse Rezende Alves ${ }^{\mathrm{VI}}$}

Resumo: Objetivo: descrever a percepção de pessoas com hipertensão arterial sobre aspectos que facilitam e dificultam a adesão ao tratamento. Método: estudo qualitativo descritivo desenvolvido com 16 pessoas atendidas em consultas de enfermagem. Realizaram-se entrevistas com roteiro semiestruturado e utilizou-se a técnica de análise de conteúdo. Os dados foram coletados entre os meses de janeiro e agosto de 2017. Resultados: observou-se que ter força de vontade, apoio familiar e multiprofissional, conhecimento sobre a patologia e formas de prevenção e medo da morte facilitou a adesão; por outro lado, pouco conhecimento, preguiça, falta de infraestrutura urbana e condições climáticas, hábito de consumir alimentos não saudáveis, bebidas alcoólicas e tabaco, custo do tratamento e esquecimento de tomar a medicação dificultaram a adesão. Conclusão: o tratamento da hipertensão acarreta mudanças na dinâmica da vida para as quais é fundamental ter força de vontade, apoio familiar e profissional, além de superar hábitos não saudáveis.

Descritores: Enfermagem; Hipertensão; Cooperação do Paciente; Cooperação e Adesão ao Tratamento; Adesão à Medicação

\footnotetext{
${ }^{\text {I }}$ Enfermeiro. Especialista em Gestão de Saúde Pública pela Faculdade Batista de Minas Gerais. E-mail: oliveira.pauloroberto@yahoo.com.br ORCID: https://orcid.org/0000-0002-0805-2249

II Enfermeira. Residente em Saúde do Adulto. Especialista em Urgência e Trauma pela Universidade Federal do Triângulo Mineiro (UFTM), Uberaba, MG, Brasil. E-mail: deboraosacramento18@gmail.com ORCID: https://orcid.org/0000-0001-6448-8330

III Enfermeira. Mestre em Saúde Coletiva. Docente do Departamento de Medicina e Enfermagem da Universidade Federal de Viçosa (UFV), Viçosa, Minas Gerais, Brasil. E-mail: flaviabatista@ufv.br ORCID: https://orcid.org/0000-0003-2360-3026

IV Enfermeira. Doutora em Enfermagem. Docente do Departamento de Medicina e Enfermagem da Universidade Federal de Viçosa (UFV), Viçosa, Minas Gerais, Brasil, E-mail: luana.toledo@ufv.br, ORCID: http://orcid.org/0000-0001-9527-7325

V Enfermeiro. Mestre em Enfermagem. Docente da Universidade Federal do Tocantins (UFT), Palmas, Tocantins, Brasil. E-mail: renansallazar@gmail.com ORCID: https://orcid.org/0000-0001-5140-4046

VI Enfermeira. Doutora em Enfermagem. Docente do Departamento de Medicina e Enfermagem da Universidade Federal de Viçosa (UFV), Viçosa, Minas Gerais, Brasil, E-mail: katiusse@ufv.br ORCID: https://orcid.org/0000-0003-3393-8567
} 
Percepção de pessoas com hipertensão arterial sobre aspectos que influenciam a adesão ... 2

\begin{abstract}
Objective: to describe the perception of people with arterial hypertension of the aspects that facilitate and hinder treatment adherence. Method: descriptive qualitative study developed with 16 people met in Nursing consultations. Interviews were conducted with a semi-structured guide and the content analysis technique was used. Data were collected between January and August 2017. Results: having willpower, family and multidisciplinary support, knowledge about the pathology and forms of prevention and fear of death facilitated adherence; on the other hand, little knowledge, laziness, lack of urban infrastructure and climatic conditions, habit of consuming unhealthy foods, alcoholic beverages and tobacco, cost of treatment and forgetfulness of taking the medication hindered adherence. Conclusion: the treatment of hypertension causes changes in the dynamics of life, being essential to have willpower, family and professional support, in addition to overcoming unhealthy habits.

Descriptors: Nursing; Hypertension; Patient Compliance; Treatment Adherence and Compliance; Medication adherence
\end{abstract}

Resumen: Objetivo: describir la percepción de las personas con hipertensión arterial sobre los aspectos que facilitan y dificultan la adherencia al tratamiento. Método: estudio cualitativo descriptivo desarrollado con 16 personas atendidas en consultas de enfermería. Se realizaron entrevistas con un guion semiestructurado y se utilizó la técnica de análisis de contenido. Los datos se recopilaron entre enero y agosto de 2017. Resultados: se observó que tener fuerza de voluntad, apoyo familiar y multidisciplinario, conocimiento sobre la patología y las formas de prevención y miedo a la muerte facilitaba la adherencia; por otro lado, poco conocimiento, pereza, falta de infraestructura urbana y condiciones climáticas, hábito de consumir alimentos no saludables, bebidas alcohólicas y tabaco, costo de tratamiento y olvido de tomar el medicamento obstaculizaba la adherencia. Conclusión: el tratamiento de la hipertensión provoca cambios en la dinámica de la vida para los que es esencial contar con fuerza de voluntad, apoyo familiar y profesional, además de superar hábitos poco saludables.

Descriptores: Enfermería; Hipertensión; Cooperación del Paciente; Cumplimiento y Adherencia al Tratamiento; Cumplimiento de la Medicación

\title{
Introdução
}

A hipertensão arterial (HA) é uma condição crônica de natureza multifatorial e silenciosa. Cursa com comprometimento do equilíbrio dos mecanismos vasodilatadores e vasoconstritores e seu diagnóstico é feito a partir de níveis pressóricos elevados e sustentados maiores ou iguais a 140 x 90 mmHg. ${ }^{1}$ A prevalência global dessa patologia é aproximadamente de um bilhão. ${ }^{2}$ Estima-se que no Brasil 32,5\% de indivíduos adultos e mais de $60 \%$ de idosos apresentem $\mathrm{HA}^{3}$ Ela é o principal fator de risco para a mortalidade por todas as causas e, principalmente, para doenças cardiovasculares (DCV), contribuindo direta ou indiretamente na carga global das doenças e nos anos de vida perdidos por incapacidades. ${ }^{1-2}$

Os principais fatores de risco para a HA são obesidade, resistência à insulina, consumo aumentado de bebidas alcoólicas e sal, idade avançada, sedentarismo, estresse e baixo consumo 
3 | Miranda PRO, Sacramento DO, Diaz FBB, Toledo LV, Pereira RSF, Rezende-Alves K

de potássio e cálcio. O tratamento inclui medidas farmacológicas, baseadas no uso de antihipertensivos e/ou não farmacológicas, como alimentação saudável, atividade física, aumento da ingestão de potássio e cálcio, controle do peso corporal, diminuição do consumo de sal e bebidas alcoólicas e abandono do hábito de fumar., ${ }^{1,4}$

A HA permanece como uma condição de difícil controle epidemiológico especialmente devido à baixa adesão dos pacientes ao tratamento a médio e longo prazo, que ocorre em cerca de $50 \%$ dos casos. $^{5-6}$ A adesão é afetada por condições socioeconômicas (baixa renda e escolaridade, dificuldade de acesso aos serviços de saúde, custo de medicamentos) e por fatores relacionados à própria doença, à terapia e ao sistema e equipes de saúde (distribuição inadequadas de medicamentos, escassez de treinamento das equipes sobre gerenciamento de doenças crônicas). ${ }^{6} \mathrm{O}$ controle eficaz da HA depende também da compreensão do paciente sobre a sua condição clínica e seu tratamento. ${ }^{6-7}$

Entretanto, pouco sucesso tem-se alcançado em termos de adesão efetiva e contenção do avanço da HA fazendo com que ela permaneça como importante problema de saúde pública. ${ }^{6,8}$ Portanto, frente magnitude da HA, das doenças crônicas não transmissíveis (DCNT) no contexto atual e da complexidade que envolve o fenômeno da adesão, aprofundar a compreensão sobre a forma de pensar e agir dos indivíduos, contribuirá para maior detalhamento e conhecimento das facilidades e barreiras encontradas para seguir o tratamento da HA.

Nesse contexto, a questão de pesquisa que norteia este estudo é: quais os aspectos facilitam e quais os que dificultam a adesão ao tratamento da HA a partir da percepção de pessoas com HA? Para responder a esses questionamentos, objetivou-se descrever a percepção de pessoas com HA sobre aspectos que facilitam e dificultam a adesão ao tratamento. 
Percepção de pessoas com hipertensão arterial sobre aspectos que influenciam a adesão ...| 4

\section{Método}

Trata-se de um estudo descritivo com abordagem qualitativa realizado com pessoas com HA cadastradas em um programa de prevenção do risco cardiovascular vinculado a uma instituição pública de ensino superior da região sudeste do Brasil. O programa surgiu em 2003, como projeto de extensão universitária, com objetivo de prestar atendimentos dietoterápicos para servidores e familiares, além de estudantes que apresentassem fatores de risco cardiovascular. Tal iniciativa se deu devido ao aumento da ocorrência de DCNT como hipertensão, dislipidemias, diabetes e obesidade, que juntas são conhecidas como síndrome metabólica e estão associadas à alta mortalidade por DCV no Brasil.

Atualmente o programa funciona em um serviço ambulatorial criado pela universidade. Ele oferece atendimentos individuais de Enfermagem, Nutrição e Educação Física e realiza atividades educativas individuais e coletivas, a fim de contribuir para a melhoria do autocuidado e do padrão alimentar; bem como, sensibilizar os pacientes cadastrados para a adoção de um estilo de vida saudável. O acompanhamento é realizado por uma equipe composta por estudantes, professores e profissionais das referidas áreas.

Para realizar este estudo foram consultados os registros de todos os 115 atendimentos de Enfermagem realizados no ano de 2016. Foram elegíveis 52 pacientes que atendiam aos seguintes critérios de inclusão: ter diagnóstico de HA e ter comparecido, regularmente, às consultas de Enfermagem no ano de 2016. Como critério de exclusão, adotou-se a presença de qualquer limitação que os impedisse de responder ao pesquisador. Do total dos pacientes, 16 foram entrevistados, pois com esse número de entrevistas atingiu-se a saturação teórica dos dados. A escolha desses participantes ocorreu de forma aleatória.

A coleta de dados aconteceu entre janeiro e agosto de 2017. O convite para participar foi feito por contato telefônico ou, pessoalmente, quando os pacientes compareciam ao serviço para 
5 | Miranda PRO, Sacramento DO, Diaz FBB, Toledo LV, Pereira RSF, Rezende-Alves K

os atendimentos. Utilizou-se um roteiro de entrevista semiestruturada elaborado pelos pesquisadores composto por duas partes: uma com questões sociodemográficos e a outra com perguntas sobre o que entendiam por HA e quais as facilidades e dificuldades encontradas para aderir ao tratamento farmacológico e não farmacológico (dieta, atividade física, redução do consumo de bebidas alcoólicas e de cigarros). Além disso, foi aplicado o teste Morisky-Green para avaliar a adesão ao tratamento medicamentoso. ${ }^{9}$

As entrevistas foram agendadas de acordo com a disponibilidade dos participantes, tiveram duração média de 25 minutos e foram gravadas em gravador digital após a anuência dos entrevistados. Posteriormente, as falas foram transcritas na íntegra, a fim de possibilitar a análise e interpretação fidedignas dos resultados. O término da coleta deu-se após ser identificada a saturação teórica dos dados, ou seja, a inclusão de novos participantes deixa de ser necessária devido à repetição das informações.

Para a análise foi utilizada a Técnica de Análise de Conteúdo, que consiste em três fases: a pré-análise, a exploração do material e o tratamento dos resultados. A primeira, destina-se à organização do material e envolve quatro etapas: leitura flutuante, que é o estabelecimento de contato com os documentos da coleta de dados; a escolha do que será analisado; a formulação das hipóteses e dos objetivos; a organização destas hipóteses ou objetivos com base nos recortes de falas que se repetem com muita frequência. Esta última etapa irá determinar os temas que orientarão a interpretação e inferência. $\mathrm{Na}$ segunda fase, são realizadas a codificação, a escolha das unidades de registro, a classificação de acordo com os temas e a categorização, ou seja, a reunião de maior número de temas correlacionados. Por fim, no tratamento dos resultados, são feitas inferências e interpretações do conteúdo manifesto e latente dos dados. ${ }^{10}$

As diretrizes éticas estabelecidas pela Resolução 466/2012 foram respeitadas, a pesquisa foi aprovada pelo Comitê de Ética e Pesquisa da Instituição pesquisada (Parecer n⿳ 1.821 .610 aprovado em 16/11/16) e todos os participantes assinaram o Termo de Consentimento Livre e 
Percepção de pessoas com hipertensão arterial sobre aspectos que influenciam a adesão ...| 6

Esclarecido. Para fins de sigilo os participantes foram codificados com a letra E, seguida do número de sequência das entrevistas.

\section{Resultados e discussão}

Participaram do estudo 16 pessoas, sendo dez mulheres $(62,5 \%)$ e seis homens $(37,5 \%)$ com idades que variaram entre 40 e 70 anos. Os participantes, na maioria, tinham mais de 60 anos $(43,7 \%)$, eram brancos $(62,5 \%)$, casados $(81,2 \%)$ e com ensino fundamental completo $(62,5 \%)$. As profissões de nível fundamental (porteiro, auxiliar de agropecuária e do lar) foram as mais frequentes, a renda familiar foi de três a quatro salários mínimos (salário mínimo em 2017- R\$ $937,00)$ e oito entrevistados $(50 \%)$ residia com três pessoas. Além de HA, 14 participantes $(87,5 \%)$ relataram outras patologias, como diabetes mellitus, dislipidemia, cardiopatia e três tiveram complicações anteriores, como Infarto Agudo do Miocárdio (IAM), Acidente Vascular Encefálico (AVE) e lesão renal.

Quanto aos hábitos de vida, oito entrevistados (50\%) praticavam, no mínimo, 150 minutos de alguma atividade física por semana; já os demais, eram sedentários. Em relação ao consumo alimentar, a maioria $(68,7 \%)$ relatou evitar: o consumo de temperos industrializados com alto teor de sódio, o uso do saleiro na mesa, temperar os alimentos com muito sal e o consumo de produtos embutidos e enlatados. Nenhum entrevistado fumava, sete declararam ser ex-fumantes $(43,7 \%)$ e três $(18,7 \%)$ consumiam bebidas alcoólicas.

O perfil dos participantes mostrou que, em geral, eram pessoas com idade mais avançada, baixa escolaridade, possuíam outras DCNT, além de alguns já terem tido complicações da HA. Tais fatores associados contribuem, substancialmente, para a ocorrência da HA e de suas complicações, IAM, AVE e insuficiência cardíaca e renal e até mesmo da morte. ${ }^{8}$ A Organização Mundial da Saúde (OMS) preconiza intervenção em fatores de risco modificáveis, a fim de reduzir as altas taxas de prevalência de HA e DCNT. Para tanto, é indispensável ter um estilo de 
7 | Miranda PRO, Sacramento DO, Diaz FBB, Toledo LV, Pereira RSF, Rezende-Alves K

vida saudável que inclua o aumento do consumo de frutas e verduras e legumes e a redução do consumo excessivo de sal, do sobrepeso e da obesidade, do uso nocivo de álcool e da inatividade física. Para ocorrer tais mudanças são necessários o fortalecimento das políticas e dos sistemas de saúde; sobretudo, o de atenção básica, mas também a conscientização das pessoas com HA acerca da importância da prevenção e do autocuidado..$^{7-8,11}$

Neste estudo, as entrevistas foram analisadas, a fim de identificar as percepções das pessoas com HA sobre os aspectos que facilitam e dificultam a adesão ao tratamento. A partir dessa análise, emergiram duas categorias: 1) aspectos que facilitam a adesão e 2) aspectos que dificultam a adesão. Cada uma traz as percepções dos participantes sobre a prática de atividades físicas, o consumo alimentar, o uso de bebidas alcoólicas e cigarro e o tratamento farmacológico.

\section{Aspectos que facilitaram a adesão}

Os entrevistados identificaram vários aspectos que potencializaram a adesão ao tratamento da HA. Dentre eles foram destacados a infraestrutura urbana para a prática de atividades físicas, o suporte comunitário, familiar e dos profissionais de saúde, a vontade de viver uma vida mais saudável e compreensão da necessidade do tratamento.

No que tange à atividade física, a companhia de amigos e familiares durante as práticas, a inserção em grupos de atividades da terceira idade, as caminhadas e o uso da bicicleta como meio de transporte contribuíram para os participantes se manterem ativos fisicamente. Além disso, foi salientado o papel preponderante da força de vontade na adoção de um estilo de vida mais saudável. Outro aspecto destacado de maneira positiva foi a existência de locais adequados e seguros próximos à residência, como enunciado nos relatos, a seguir.

O local que moro facilita muito, tem uma pista para caminhar. (E13)

Grupo da terceira idade, lá eu me sinto bem melhor, gosto muito. É uma hora que a gente distrai bastante, por causa das colegas. (E5) 
Percepção de pessoas com hipertensão arterial sobre aspectos que influenciam a adesão ...| 8

A caminhada que faço todos os dias é do colégio da minha filha até o local que pego a van para ir ao serviço. Estabeleci isso: ir a pé para não ficar sedentária. [...] e a única maneira que encontrei foi essa. Sei também que, para controlar minha pressão e meu colesterol, precisava de atividade física. (E9)

A caminhada é uma coisa gostosa, vai eu e mais dois vizinhos um anima o outro, é a hora que batemos papo, distrai e é gostoso. (E14)

O fato de eu ir trabalhar de bicicleta. Foi uma decisão que tomei porque não estava conseguindo controlar a hemoglobina glicada. Eu andava de moto, aí passei a andar de bicicleta. (E1)

O que facilita? A força de vontade mesmo. (E7)

O aumento da atividade física, sobretudo no lazer, está associado à diminuição da PA e de fatores de risco para a HA, como o acúmulo de tecido adiposo abdominal e visceral. ${ }^{12} \mathrm{~A}$ existência de condições ambientais e infraestrutura que proporcionem alguma facilidade, a proximidade a locais apropriados à prática de atividade física, a percepção do estado de saúde e a força de vontade são aspectos que contribuem para uma vida mais ativa fisicamente e melhor controle da PA.,13

Somado a isso, ter a companhia de pessoas da família/comunidade estimula a vontade de se exercitar. A prática, realizada em grupo, pode promover a interação social, construir e fortalecer amizades, além de um membro do grupo incentivar outro, potencializando seu empenho no autocuidado. ${ }^{14}$ Outro aspecto facilitador foi a utilização do percurso para o trabalho ou para levar filhos à escola para abandonar o sedentarismo. Atitudes como estas têm sido tendência, principalmente, na população economicamente ativa que troca os veículos por caminhada, bicicleta ou até corrida com o objetivo de alcançar melhor qualidade de vida e de saúde. ${ }^{8}$

Em relação ao consumo alimentar, o suporte familiar e profissional, a força de vontade, a motivação, o respeito pelas preferências alimentares e a substituição do sal por ervas aromáticas 
9 | Miranda PRO, Sacramento DO, Diaz FBB, Toledo LV, Pereira RSF, Rezende-Alves K

foram elencados pelos participantes como pontos que favorecem a adesão à dieta. Os relatos a seguir evidenciam tais aspectos.

Meu filho e marido sempre estão me cobrando seguir tudo que a nutrição e o médico passam e compram coisas da dieta. (E12)

As pessoas confundem que bem temperado tem que ser muito salgado. Bem temperado é você saber escolher os tipos de temperos certos, os tipos de ervas que vão dar sabor e o pessoal quer entupir de sal e o sal, pelo contrário, até rouba o sabor. (E2)

A força de vontade. Depois que você percebe como nosso organismo melhora com uma boa alimentação, a força de vontade aumenta. (E9)

A dieta foi feita com aquelas coisas que gosto. (E13)

Vir na nutricionista ajuda demais seguir a dieta. Ela sempre tira dúvidas e fala tudo que faz bem e mal; isso ajuda demais, porque eu e minha esposa não temos muito estudo para conhecer essas coisas. (E15)

Está bem estabelecido que padrões alimentares ricos em frutas, verduras e legumes, grãos integrais, lácteos com baixo teor de gorduras saturadas e baixo teor de sódio estão associados à redução da PA. ${ }^{15}$ Além disso, o consumo desse tipo de dieta favorece a ingestão de componentes bioativos, relacionados com uma diminuição da PA, como as classes dos flavonoides, presentes em frutas, hortaliças, em alguns chás, no vinho e no cacau, e das antocianinas, encontradas em frutas e hortaliças de cor avermelhada (como açaí, uva, maçã, morango, cereja, acerola e repolho-roxo) e de micronutrientes como o potássio e o cálcio. ${ }^{1,16}$

A mudança na alimentação pode ocorrer com a inserção gradativa de alimentos com efeito protetor e que sejam palatáveis. Além disso, quando a família apoia a pessoa com HA e opta por escolhas alimentares saudáveis, ela incentiva a incentiva na adesão ao plano alimentar a longo prazo. $^{14,17}$ Da mesma forma, o aconselhamento nutricional pode potencializar a adesão ao fornecer informações sobre os tipos de alimentos que devem ser consumidos e evitados. ${ }^{18}$ 
Percepção de pessoas com hipertensão arterial sobre aspectos que influenciam a adesão ... 10

Quanto ao consumo de bebidas alcoólicas, foi mencionado que não possuir o hábito de beber, exageradamente, ou tomar sucos, água e refrigerantes como alternativa aos etílicos os ajudaram no tratamento da HA. Destaca-se também que a vontade de manter ou de melhorar a saúde, o medo de alterar a PA e de morrer e as orientações recebidas dos profissionais de saúde impulsionaram as mudanças de comportamento, conforme os depoimentos a seguir.

É questão de costume mesmo, nunca tive vício, meu pai repreendia, então a gente foi policiando quanto a isso. (E1)

Quando estou em festas ou local com gente bebendo se tem o suco, água e até mesmo um pouco de refrigerante, eu uso e passa a vontade. Não bebo cerveja, que tenho medo de alterar a pressão. (E5)

Comecei a tomar remédio de pressão, não posso ficar bebendo. Fui orientada a trocar a bebida por chás e usar algumas ervas, então minhas dores de cabeça melhoraram muito, foi aí que percebi que a bebida estava fazendo mal. (E9)

Como fiquei muito doente e fui internado, o médico disse que se eu tomasse qualquer bebida alcoólica poderia até morrer. (E14)

O consumo de bebida alcoólica, culturalmente, é tido como um agente de interação social, que promove alegria e diversão e, para homens, associa ao exercício da masculinidade. Entretanto, o consumo exagerado torna o indivíduo mais vulnerável a situações de risco à saúde, violência e acidentes. ${ }^{19}$ Usuários de bebidas alcoólicas têm duas vezes mais chance de não aderir ao tratamento de HA. Além disso, o consumo habitual associa a elevação a PA de forma linear. ${ }^{1}$

A percepção dos participantes desta pesquisa foi que a persistência, a vontade, o estabelecimento de metas, o apoio familiar e o auxílio de medicações os ajudaram a abandonar o cigarro.

Fumei por muitos anos, esposa sempre cobrando parar, eu tentava e não conseguia. Quando meu filho nasceu comecei a não fumar dentro de casa e fui reduzindo até parar. (E14) 
11 | Miranda PRO, Sacramento DO, Diaz FBB, Toledo LV, Pereira RSF, Rezende-Alves K

Nos primeiros dias foram difíceis então evitei sair, beber café, tomar cerveja e encontrar com amigos. (E2)

Fiz o tratamento, tomei remédio, usei adesivo. Meus filhos e meu marido ajudaram muito. (E11)

Estabeleci a data que ia parar, o dia que fizesse 50 anos. Como eu estava sabendo que faltava um mês, uma semana, um ano ou um dia eu apaguei a ideia do cigarro. (E2)

A cessação do tabagismo associa a menor risco cardiovascular, entretanto, seus efeitos na redução da PA não estão totalmente claros. Sabe-se que interromper o uso do cigarro é benéfico para o controle da PA e para o uso regular dos anti-hipertensivos, melhorando o prognóstico das pessoas com HA. ${ }^{1}$

Parar de fumar é uma decisão difícil e demanda tempo para ser concretizada. Além de motivações extrínsecas, como o apoio e incentivo familiar, a pessoa necessita encontrar razões que fortaleçam sua motivação intrínseca, como o nascimento do filho e o estabelecimento de uma data. Além disso, o suporte psicológico e tratamento medicamentoso associados aumentam as possibilidades de sucesso. ${ }^{20}$

Os participantes também relataram que reconhecer que o hábito era nocivo à saúde serviu de estímulo para parar de fumar. Da mesma forma, viver a experiência do próprio adoecimento, ou de pessoas próximas, ajudou na conscientização dos prejuízos desencadeados pelo fumo. Os depoimentos a seguir sintetizam tais ideias.

É mesmo pela pressão alta. O médico falou para mim que o fumo é muito prejudicial para todo mundo e, principalmente, para quem tem problema de pressão. (E7)

Meu pai era fumante e cheirador de rapé (fumo consumido ralado através da administração pela mucosa nasal), acabou morrendo com problemas 
Percepção de pessoas com hipertensão arterial sobre aspectos que influenciam a adesão ...| 12

pulmonares. Ver o que ele passou estimulou a parar de fumar e, na mesma época, adoeci com pneumonia e isso forçou a barra e eu parei. (E1)

A nicotina começou a prejudicar meus dentes e eu tive que fazer um tratamento dentário. Tive que fazer implantes. Se não colaborar vou perder um tratamento muito caro. (E2)

A existência de problemas de saúde e encargos financeiros e o fato de compartilhar as experiências de adoecimento de outras pessoas são aspectos que fortalecem a decisão pela interrupção do hábito de fumar. ${ }^{20}$ Por vezes, o aparecimento de doenças ou hospitalizações por problemas relacionados ao fumo constitui um marco para a cessação. Nesse caso, essa atitude traz implícita a preocupação em ter boas condições de saúde e aumentar a sobrevivência tornando a pessoa mais receptiva a mudanças de hábito. ${ }^{21}$

Por fim, a adesão ao tratamento farmacológico foi avaliada através da aplicação da escala de Morisk-Green. ${ }^{9}$ Os resultados mostraram que oito eram aderentes e oito como não aderentes. Os participantes retrataram que para ajudá-los a lembrar de tomar a medicação usavam lembretes e aparelhos sonoros. Pontuaram também que a vontade, a alegria de viver, o apoio familiar e o medo de complicações os motivaram a cuidar mais da saúde. Os relatos a seguir reúnem tais aspectos:

Força de vontade de querer seguir tudo certo, medo de piorar minha saúde e de morrer. Gosto demais de viver, cuido da minha saúde para conseguir fazer tudo que gosto [...]. Meu marido fica sempre perguntando se já tomei o remédio quando chega no horário. (E13)

Eu ponho na minha mesa de cabeceira, então eu acordo e tomo, porque é a primeira coisa que vejo. (E2)

Colocava o despertador no horário de tomar o medicamento. (E6)

A adesão ao tratamento medicamentoso da HA é essencial para a redução da morbimortalidade por DCV. O medo de agravos à saúde e atitudes positivas, como alegria e 
13 | Miranda PRO, Sacramento DO, Diaz FBB, Toledo LV, Pereira RSF, Rezende-Alves K

prazer de viver, contribuem para aumentar a disposição em utilizar os medicamentos. ${ }^{22} \mathrm{~A}$ percepção de apoio e a participação familiar ativa no tratamento fornecem suporte e estímulo para o enfrentamento das alterações impostas pelas doenças. ${ }^{17}$ Além disso, quando a pessoa com HA reconhece a necessidade e aceita o tratamento, ela cria estratégias simples e eficazes para evitar o uso inadequado e/ou esquecimentos. ${ }^{7}$

Vale ressaltar que que a adesão ao tratamento deve ser vista como um fenômeno multidimensional. Ela envolve questões subjetivas como hábitos, crenças e conhecimento, mas também aspectos referentes ao sistema de saúde, como o acesso aos serviços e medicamentos e à terapia, como efeitos colaterais. Nesse contexto, a pessoa com HA encontra desafios para conduzir adequadamente o tratamento e não deve ser considerada a única responsável quando ocorre fracasso. ${ }^{6}$

\section{Aspectos que dificultaram a adesão}

Nesta pesquisa, constatou-se que os participantes sabiam pouco sobre a patologia e formas de prevenção. Eles relacionaram a HA a sintomas, à cronicidade e ao risco de complicação e morte:

É quando a gente sente dor de cabeça e as pernas ficam inchadas. (E8)

Não sei, o médico disse que eu tinha isso e era para sempre. (E14)

É um problema no coração que pode matar. (E12)

Identificar o conhecimento do paciente representa uma etapa inicial do acompanhamento. O pouco conhecimento pode levar a não percepção da gravidade e do risco de complicações, ao desinteresse pelo problema e, consequentemente, à baixa adesão.,11 Portanto, investir no processo educativo tem impacto na promoção da saúde de pessoas com HA podendo favorecer mudanças de hábitos de vida, escolhas mais conscientes e saudáveis, bem como a redução do risco cardiovascular. ${ }^{11}$ 
Percepção de pessoas com hipertensão arterial sobre aspectos que influenciam a adesão ...| 14

Em relação à prática de atividades físicas, os participantes citaram a preguiça, a falta de tempo por estar trabalhando, as condições climáticas desfavoráveis e desconfortos físicos decorrentes de alguma patologia como principais dificultadores. Os depoimentos a seguir sumarizam tais temas:

A dificuldade é a própria preguiça. (E1)

Hoje, o que dificulta é meu trabalho. Levanto cedo, venho para o trabalho e, à tarde, quando chego, quero tomar um banho e descansar, então falta horário para fazer alguma coisa. (E3)

É porque tenho dor na perna. (E4)

A arritmia que eu tenho no coração, sinto muita alteração no coração por causa dessa arritmia. (E5)

O frio incomoda um pouco. (E10)

São considerados sedentários, adultos que não praticam pelo menos 150 minutos de atividade física moderada por semana. ${ }^{1}$ A superação do sedentarismo e da acomodação impõe mudanças na dinâmica de vida que nem sempre são acolhidas com facilidade pela pessoa com HA; sobretudo, pelas que não possuem o hábito de se exercitar. ${ }^{23}$

Para as pessoas ativas fisicamente, as condições climáticas desfavoráveis podem fazê-las reduzir ou interromper as atividades, fato mais evidenciado no inverno e em regiões com climas instáveis. Em relação àquelas que trabalham, as demandas, os horários e a sobrecarga podem resultar em um menor tempo dedicado ao cuidado à saúde. Por fim, patologias, dores e desconfortos acabam contribuindo para a inatividade física. ${ }^{13,23}$

No tocante à dieta, os participantes relataram que o que mais dificultou o cumprimento das recomendações foi o hábito de consumir alimentos não saudáveis ou preparados por terceiros, a participação em festas, a alimentação fora de casa, o pouco conhecimento sobre o 
15 | Miranda PRO, Sacramento DO, Diaz FBB, Toledo LV, Pereira RSF, Rezende-Alves K

que comer e sobre os efeitos da dieta na HA. Aliado a isto, alguns enfrentaram a resistência da família em se abster do excesso de sal.

Problema é que nós temos uma empregada doméstica, às vezes, ela erra nas dosagens. Sábado e domingo a gente costuma almoçar em restaurantes, você não controla se o cara lá atolou no tempero. (E2)

Fui acostumada, desde criança, a comer muito alimento gorduroso, temperado e uns industrializados. (E6)

Eu que faço a comida lá em casa. Se fizer sem sal e seguindo só a dieta acho que ninguém come. Eles não precisam comer comida sem sal por minha causa. Uma vez que tentei cortar o sal, eles reclamaram. (E14)

É saber as comidas que não posso. Estava comendo umas coisas achando que não fazia mal. Quando falei com a nutricionista, ela cortou, disse que aumenta a pressão. (E15)

O apoio familiar pode contribuir para que a pessoa com HA realize mudanças na alimentação e aceite melhor a necessidade de redução do sal e as alterações nos sabores. ${ }^{17,24} \mathrm{Na}$ dieta feita em casa podem ser priorizados alimentos in natura e minimamente processados em detrimento dos industrializados/ultraprocessados, o que representa uma forma de melhorar a qualidade do que é consumido e tem sido associado a melhores resultados dos níveis pressóricos. ${ }^{25}$

Comer fora de casa torna mais difícil seguir a dieta recomendada. A oferta de alimentos industrializados, ricos em sal, gorduras saturadas e açúcar livre, tipo fast foods, é maior e não é possível ter controle da quantidade desses nutrientes em alimentos preparados por terceiros. A participação em festas também representou uma barreira, pois nestes ambientes há grande disponibilidade de alimentos não saudáveis, bebidas açucaradas, como refrigerantes e sucos industrializados, e alcoólicas tornando mais árduo o seguimento da dieta. Ademais, a alimentação tem um papel cultural e social que sobrepuja o da nutrição. ${ }^{11}$ 
Percepção de pessoas com hipertensão arterial sobre aspectos que influenciam a adesão ...| 16

Não é incomum pessoas com HA possuírem conhecimento insuficiente sobre a quantidade recomendada de sódio por dia ( $2 \mathrm{~g}$, ou seja, $5 \mathrm{~g}$ de cloreto de sódio), além de dificuldades para compreender as informações presentes nos rótulos. Conhecer os alimentos que devem ser evitados e entender o quanto o excesso de sal altera o mecanismo fisiológico pode contribuir para a prevenção da HA e de complicações. ${ }^{24}$

No que concerne ao consumo de bebidas alcoólicas é conhecido que ele favorece a interação social, proporciona o bem-estar e causa prazer tornando mais difícil sua redução. Os depoimentos a seguir ilustram as percepções dos participantes sobre tais pontos.

Era o prazer de beber, eu não bebia em casa, saía para descontrair e curtir alguma coisa e a cerveja estava naquele momento me fazendo bem, era o prazer daquele momento, o bem-estar social. (E2)

Tomar cerveja é um hobby, sentar num bar, tomar uma cerveja, vou para o forró tomo minha cerveja, então vou sem beber nada? Aí fico desanimado, quando bebo fico mais animado, mais enérgico e sinto melhor. (E7)

Tinha divorciado então queria aproveitar a vida, sair com as amigas, divertir, beber. (E9)

Os relatos evidenciam que a representação social do consumo de bebidas alcoólicas é permeada por crenças positivas sobre seu papel socializador/integrador. Neste contexto, são utilizadas, sobretudo por homens, para entreter e favorecer relacionamentos interpessoais. Todavia, essas pessoas são expostas ao maior risco de dependência e ao aparecimento de doenças. ${ }^{19}$

O consumo habitual e excessivo de álcool associa positivamente ao aumento da PA e à incidência de HA. Portanto, recomenda-se que não deva ultrapassar uma dose (14g de etanol que equivale a $350 \mathrm{ml}$ de cerveja, $150 \mathrm{ml}$ de vinho ou $45 \mathrm{ml}$ de bebida destilada) para as mulheres e pessoas com baixo peso e duas doses para homens. ${ }^{1}$ Quanto ao cigarro, os participantes reconheceram que o hábito de fumar gera dependência, mas também relataram que 
17 | Miranda PRO, Sacramento DO, Diaz FBB, Toledo LV, Pereira RSF, Rezende-Alves K

proporcionava prazer e alívio do estresse. Ademais, ao interromperem o uso, precisaram lidar com gatilhos de recaídas, tais como os ambientes com pessoas fumando e as alterações emocionais cotidianas. Esses elementos estão presentes nos depoimentos.

A dificuldade foi a dependência, se não tiver uma força de vontade grande, não para. Quando traga você relaxa ou acha que está relaxando, mas o cigarro não é inimigo, ele é amigo, a cerveja não é inimiga, ela é amiga, a cerveja não é agressiva, é inofensiva, porque descontrai, relaxa: a mesma coisa, o cigarro. (E2)

Me dava nervoso e tremedeira, então tinha que fumar. (E11)

Levantava de manhã e falava 'hoje eu não vou fumar'. Quando eu pensava que não, estava fumando um maço de cigarros. No meu serviço podia fumar, os colegas todos fumando, então tudo isso dificulta. (E6)

O uso do cigarro, para muitas pessoas, ajuda a diminuir o estresse cotidiano, sobretudo quando acreditam que fumar traz tranquilidade e auxilia lidar com emoções indesejadas. ${ }^{20}$ Para de fumar constitui uma experiência difícil, por ser a dependência um fenômeno complexo que engloba aspectos comportamentais, cognitivos, sociais e fisiológicos. ${ }^{20} \mathrm{~A}$ abordagem terapêutica baseada na combinação de intervenções cognitivas com treinamento de habilidades comportamentais e/ou educativas associadas à farmacoterapia (reposição de nicotina e bupropiona) são recursos que aumentam o sucesso no tratamento..$^{20-21}$

Em relação à adesão ao tratamento farmacológico, as principais dificuldades citadas foram esquecimento, custo dos medicamentos, déficit visual e efeitos indesejados do tratamento. Tais considerações podem ser identificadas nos depoimentos.

$\grave{A} s$ vezes, tinha que sair e esquecia de tomar naquele dia. (E6)

Como era estudante, vivia com uma bolsa para sustentar eu e minha filha, então tinha dificuldade em comprar o medicamento. (E9) 
Percepção de pessoas com hipertensão arterial sobre aspectos que influenciam a adesão ...| 18

Minha dificuldade é saber qual comprimido tenho que tomar de manhã e qual tenho que tomar à noite, não estou enxergando direito e não consigo ler. (E14)

\section{O primeiro medicamento que eu fiz uso deu um efeito colateral. (E2)}

\section{Ah! Eu acho chato isso, mas tenho que tomar. (E11)}

A condição socioeconômica pode influenciar, negativamente, na adesão ao tratamento da HA. Pessoas com renda familiar mais baixa podem ter mais dificuldades de acesso aos serviços de saúde e aos medicamentos. Neste sentido, o Sistema Único de Saúde (SUS) tem garantido o acesso gratuito, entretanto, ainda há necessidade de fortalecer as políticas públicas de redução das desigualdades de acesso a medicamentos no país. ${ }^{22}$

Ressalta-se que por si só o acesso não garante bons resultados, pois muitas pessoas com HA podem ter dificuldades com o manejo do tratamento. O número de medicamentos utilizados está diretamente associado ao esquecimento, o uso de três ou mais aumenta a chance de não recordar. Portanto, quando é necessária a combinação de fármacos, é indispensável que as equipes busquem estratégias, junto aos pacientes e famílias, a fim de reduzir as taxas de esquecimento. ${ }^{22}$

O uso de maior número de medicações também pode acarretar o aparecimento de algum incomodo orgânico, levando o paciente a fazer o tratamento de forma irregular ou a abandoná-lo. Para se efetivo e facilitar a adesão, o tratamento farmacológico deve ter pouco ou nenhum efeito adverso e não interferir, negativamente, na qualidade de vida. Além disso, é comum a diminuição da acuidade visual com o aumento da idade. Esta condição pode interferir com a capacidade de ler as informações nas embalagens e de recordar as medicações, as doses e os horários de tomar. ${ }^{16,22}$

Por fim, destaca-se que a presente investigação teve como limitação o fato de ter um número pequeno de participantes e um único serviço como cenário. Assim, a complexidade das 
19 | Miranda PRO, Sacramento DO, Diaz FBB, Toledo LV, Pereira RSF, Rezende-Alves K

vivencias pode ter sido retratada parcialmente, embora ao investigar aspectos do comportamento humano encontrem-se certos padrões que tendem a se repetir.

\section{Conclusão}

Para os participantes deste estudo, a HA acarretou a necessidade de adaptação à uma nova condição. Consequentemente, exigiu a introdução de novos hábitos de vida e a busca de outros sentidos para a experiência com o alimento, a atividade física, a bebida alcoólica, o cigarro e o tratamento farmacológico. A mudança no estilo de vida decorrente de um adoecimento constitui um processo de aprendizagem marcado por progressos graduais. A força de vontade, o apoio familiar e multiprofissional, o conhecimento da condição de saúde e da importância do tratamento e o medo da morte ajudam a vivenciar esta etapa de transformações.

Entretanto, não é fácil modificar comportamentos, hábitos e crenças consolidados ao longo da vida, tampouco aprender novas formas de cuidar da saúde. O tratamento da HA também implica lidar com situações desafiadoras que precisam ser contornadas, como a desmotivação para realizar atividades físicas, a falta de infraestrutura urbana, as condições climáticas como o frio, o hábito de consumir alimentos não saudáveis, bebidas alcoólicas e cigarro, o custo de medicamentos e o esquecimento da medicação.

Tendo em vista a magnitude epidemiológica da HA, seu impacto na qualidade de vida das pessoas e que a baixa adesão ao tratamento medicamentoso e não medicamentoso afeta seus resultados, ressalta-se que os achados desta pesquisa trazem implicações práticas importantes no que se refere a importância de envolver a família no cuidado, como fonte de apoio e sustentação, para potencializar a motivação e aderência dos pacientes. Além disso, reforça que atividades educativas individuais ou coletivas realizada pelas equipes multiprofissionais 
Percepção de pessoas com hipertensão arterial sobre aspectos que influenciam a adesão ...| 20

direcionadas à promoção da saúde das pessoas com HA podem ser estratégias promissoras para ampliar o conhecimento, o empoderamento e autonomia para o autocuidado.

Vale salientar que ao investigar uma temática relevante para a Enfermagem, a saúde coletiva e a promoção da saúde, esta pesquisa pode contribuir com profissionais de saúde e, sobretudo, os de enfermagem, com informações mais aprofundadas acerca de aspectos intrínsecos (conhecimentos, motivações, desejos e crenças) e extrínsecos (família, ambiente, condições socioeconômicas) que afetam a adesão ao tratamento da HA e a escolha de comportamentos mais saudáveis para o autocuidado.

\section{Referências}

1. Sociedade Brasileira de Cardiologia. 7 ${ }^{\mathbf{a}}$ Diretrizes Brasileiras de Hipertensão Arterial. Arq Bras Cardiol [Internet]. 2016 [acesso em 2019 mar 18];107(3): Supl. 3. Disponível em: http://publicacoes.cardiol.br/2014/diretrizes/2016/05_HIPERTENSAO_ARTERIAL.pdf

2. Forouzanfar MH, Liu P, Roth GA, Ng M, Biryukov S, Marczak L, et al. Global burden of hypertension and systolic blood pressure of at least 110 to $115 \mathrm{~mm} \mathrm{Hg}$, 1990-2015. JAMA Netw Open. 2017;317(2):16582. doi: https://doi.org/10.1001/jama.2016.19043

3. Malta DC, Gonçalves RPF, Machado IE, Freitas MIF, Azeredo C, Szwarcwald CL, et al. Prevalence of arterial hypertension according to different diagnostic criteria. Rev Bras Epidemiol. 2018;21(Suppl 1): e180021. doi: https://doi.org/10.1590/1980-549720180021.supl.1

4. Oparil S, Acelajado MC, Bakris GL, Berlowitz DR, Cífková R, Dominiczak AF, et al. Hypertension. Nat Rev Dis Primers. 2018; 4:18014. https://doi.org/10.1038/nrdp.2018.14

5. Abegaz TM, Shehab A, Gebreyohannes EA, Bhagavathula AS, Elnour AA. Nonadherence to antihypertensive drugs: a systematic review and meta-analysis. Medicine (Baltimore). 2017; 96(4):1-9. https://doi.org/10.1097/MD.0000000000005641

6. World Health Organization (WHO). Adherence to long-term therapies: evidence for action [Internet]. Geneva: WHO; 2003 [cited 2019 Mar 18]. Available from: https://www.who.int/chp/knowledge/publications/adherence_full_report.pdf?ua=1

7. Santos RZ, Bonin CDB, Martins ETC, Pereira Junior M, Ghisi GLM, Macedo KRP, et al. Development and psychometric validation of HIPER-Q to assess knowledge of hypertensive patients in cardiac rehabilitation. Arq Bras Cardiol. 2017; 110(1):60-8. doi: https://doi.org/10.5935/abc.20170183 
8. World Health Organization (WHO). Global status report on noncommunicable diseases 2014 [Internet]. Geneva: WHO; 2014 [cited 2018 Jan 08]. Available from: https://www.who.int/nmh/publications/ncd-status-report-2014/en/

9. Morisky DE, Green LW, Levine DM. Concurrent and predictive validity of a self-reported measure of medication adherence. Med Care. 1986;1(24):67-74. doi: http://doi.org/10.1097/00005650-198601000-00007

10. Bardin L. Análise de conteúdo. Lisboa: Edições 70; 2011.

11. Machado JC, Cotta RMM, Moreira TR, Silva LS. Análise de três estratégias de educação em saúde para portadores de hipertensão arterial. Ciênc Saúde Colet. 2016;21(2):611-20. doi: https://doi.org/10.1590/1413-81232015212.20112014

12. Liu X, Zhang D, Liu Y, Sun X, Han C, Wang B, et al. Dose-response association between physical activity and incident hypertension. Hypertension. 2017;69(5):813-20. doi: https://doi.org/10.1161/HYPERTENSIONAHA.116.08994

13. Ribeiro AQ, Salgado SML, Gomes IS, Fogal AS, Martinho KO, Almeida LFF, et. al. Prevalence and factors associated with physical inactivity among the elderly: a population-based study. Rev Bras Geriatr Gerontol. 2016; 19(3):483-93. doi: https://doi.org/10.1590/1809-98232016019.150047

14. Smith GL, Banting L, Eime R, O'Sullivan G, Van Uffelen JGZ. The association between social support and physical activity in older adults: a systematic review. Int J Behav Nutr Phys Act. 2017; 14(1):44-9. doi: https://doi.org/10.1186/s12966-017-0509-8

15. Paula TP, Viana LV, TZ Neto A, Leitão CB, Gross JL, Azevedo MJ. Effects of the DASH diet and walking on blood pressure in patients with type 2 diabetes and uncontrolled hypertension: a randomized controlled trial. J Clin Hypertens. 2015; 17(11):895-901. doi: https://doi.org/10.1111/jch.12597.

16. Vendrame S, Klimis-Zacas D. Potential factors influencing the effects of anthocyanins on blood pressure regulation in humans: a review. Nutrients. 2019;11(6):1431-42. https://doi.org/10.3390/nu11061431

17. Bahari G, Scafide K, Krall J, Mallinson RK, Weinstein AA. Mediating role of self-efficacy in the relationship between family social support and hypertension self-care behaviours: a cross-sectional study of Saudi men with hypertension. Int J Nurs Pract. 2019; 25(6):e12785. doi: https://doi.org/10.1111/ijn.12785

18. Mahdavi R, Asl AB, Abadi MAJ, Namazi N. Perceived barriers to following dietary recommendations in hypertensive patients. J Am Coll Nutr. 2017;36(3):193-9. doi: https://doi.org/10.1080/07315724.2014.966176

19. Rosa LFA, Nascimento ARA. Representações sociais de bebida alcoólica para homens universitários. Arq Bras Psicol. 2015 [acesso em 2017 nov 20];67(1):3-19. Disponível em: http://pepsic.bvsalud.org/scielo.php?script=sci_arttext\&pid=S1809-52672015000100002\&lng=pt\&nrm=iso 
Percepção de pessoas com hipertensão arterial sobre aspectos que influenciam a adesão ...| 22

20. Jesus MCP, Silva MH, Cordeiro SM, Kortchmar E, Zampier VSB, Merighi MAB. Understanding unsuccessful attempts to quit smoking: a social phenomenology approach. Rev Esc Enferm USP. 2016; 50(1):71-8. doi: http://dx.doi.org/10.1590/S0080-623420160000100010

21. Kazemzadeh Z, Manzari ZS, Pouresmail Z. Nursing interventions for smoking cessation in hospitalized patients: a systematic review. Int Nurs Rev. 2016;64(2):263-75. doi: https://doi.org/10.1111/inr.12320

22. Gewehr DM, Bandeira VAC, Gelatti GT, Colet CF, Oliveira KR. Adesão ao tratamento farmacológico da hipertensão arterial na Atenção Primária à Saúde. Saúde Debate. 2018;42(116):179-90. doi: https://doi.org/10.1590/0103-1104201811614

23. Franco MR, Tong A, Howard K, Sherrington C, Ferreira PH, Pinto RZ, et al. Older people's perspectives on participation in physical activity: a systematic review and thematic synthesis of qualitative literature. $\mathrm{Br} \mathrm{J}$ Sports Med. 2015;49(19):1268-76. doi: http://dx.doi.org/10.1136/bjsports-2014-094015

24. Teixeira JF, Goulart MR, Busnello FM, Pellanda LC. Hypertensives' knowledge about high-sodium foods and their behavior. Arq Bras Cardiol. 2016; 106(5):404-10. doi: https://doi.org/10.5935/abc.20160049.

25. Mendonça RD, Lopes ACS, Pimenta AM, Gea A, Martinez-Gonzalez MA, Bes-Rastrollo M. Ultraprocessed food consumption and the incidence of hypertension in a Mediterranean cohort: the Seguimiento Universidad de Navarra Project. Am J Hypertens. 2016; 30(4):358-66. https://doi.org/10.1093/ajh/hpw137

Editora Científica: Tânia Solange Bosi de Souza Magnago

Editora associada: Maria Denise Schimith

\author{
Autor correspondente \\ Katiusse Rezende Alves \\ E-mail: katiusse@ufv.br \\ Endereço: Av. Peter Henry Rolfs, s/n, Campus Universitário - Viçosa, MG - CEP: 36570-900
}

\title{
Contribuições de Autoria
}

1 - Paulo Roberto de Oliveira Miranda

Concepção/planejamento do estudo/pesquisa; obtenção, análise e interpretação dos dados; redação do artigo.

\section{2 - Débora de Oliveira Sacramento}

Revisão final com participação crítica e intelectual no artigo. 
23 | Miranda PRO, Sacramento DO, Diaz FBB, Toledo LV, Pereira RSF, Rezende-Alves K

\section{3 - Flávia Batista Barbosa de Sá Diaz}

Revisão final com participação crítica e intelectual no artigo.

4 - Luana Vieira Toledo

Revisão final com participação crítica e intelectual no artigo.

\section{5 - Renan Sallazar Ferreira Pereira}

Revisão final com participação crítica e intelectual no artigo.

\section{Autor 6 - Katiusse Rezende-Alves}

Concepção/planejamento do estudo/pesquisa; obtenção, análise e interpretação dos dados; redação do artigo, revisão final com participação crítica e intelectual no artigo.

\section{Como citar este artigo}

Miranda PRO, Sacramento DO, Diaz FBB, Toledo LV, Pereira RSF, Rezende-Alves K. Percepção de pessoas com hipertensão arterial sobre aspectos que influenciam a adesão ao tratamento. Rev. Enferm. UFSM. 2021 [Acesso em: Ano Mês Dia]; vol.11 e6: 1-23. DOI:https://doi.org/10.5902/2179769242403 